\title{
Museo R. P. Gustavo Le Paige s. j., San Pedro de Atacama: Estudio de las condiciones medioambientales en un ámbito de adobes
}

FRANCISCO TÉLlEZ ${ }^{1}$

\section{RESUMEN}

Luego de dos años de investigación, período durante el cual se efectuaron sistemáticas mediciones de las condiciones medioambientales, tanto al interior como al exterior del IIAM, se ha logrado perfilar el comportamiento de las diversas unidades que conforman este museo. Tal es así que se han logrado definir con cierto grado de exactitud aquellas áreas más propicias y adecuadas para la conservación de aquellas colecciones definidas como delicadas. De la misma manera se ha logrado definir aquellos espacios que presentan más o menos deficiencias respecto de la conservación de colecciones. El comportamiento integral del edificio en relación a lo que acontece en el exterior del mismo, nos ha permitido llegar a algunas conclusiones que, aunque primarias, descubren sus cualidades como asimismo sus falencias y debilidades, conocimiento básico para planificar cualquier tipo de intervención global o puntual en su estructura.

\begin{abstract}
After two years of investigation, during which systematical measurements in relation to the environmental conditions of the interior as well as the exterior of the Instituto de Investigaciones Arqueológicas y Museo (IIAM), have been carried our, we have obtained an outline of the behaviour of the different units of the museum.To a certain degree of accurancy, we have also been able to determine those areas most propitous and suitable for the conservation of those collections defined as being most susceptible. In the same way, those areas less suitable, because of deficiencies as regards the conservation of the collections, have been defined. The property of the building, taken as a unified whole relative to what may occur in its outdoor surroundings, has made us draw some conclusions conceming its qualities as well as its lacks and weaknesses; basic knowledge to be taken into account in any planning of alteración of the structure.
\end{abstract}

\section{Introducción}

El presente trabajo es un informe preliminar de las investigaciones medioambientales al interior del Museo R. P. Gustavo Le Paige s. j., que se han

1 Instituto de Investigaciones Arqueológicas y Museo R. P. Gustavo Le Paige, s. j., Universidad Católica del Norte, San Pedro de Atacama, CHILE. venido llevando a cabo durante estos dos últimos años (mediados de 1991 a mediados de 1993). Este se ha definido como básico y prioritario en cuanto sobre él se fundamentan las acciones en materias de conservación preventiva al interior del IIAM.

Si bien es cierto esta investigación tiene como objetivo básico el de proveer de información sustentable para conocer el real comportamiento climatológico del edificio que ocupa el IIAM, es de suma importancia proseguir de manera indefinida con el "monitoreo" de dichas condiciones, con el fin de estar atentos a cualquier cambio que se suceda en este aspecto.

Muchos de los problemas detectados en estas materias están siendo confirmados con registros exactos, a través de instrumentos especializados y con una planificación coherente para llegar a un resultado confiable.

Más que pretender establecer diferencias comparativas del comportamiento climatológico a través de un espacio determinado de tiempo (mensual, anual, etc.) se pretende establecer una relación comparativa entre las diversas unidades internas del IIAM (que sabemos estructuralmente diferentes), durante un mismo período y afectos a los mismos cambios externos.

En este primer intento se ha podido perfilar un comportamiento global del edificio que podría calificarse de aceptable dadas las condiciones constructivas y lo irregular de éste, además del drástico clima local (desierto de altura) caracterizado principalmente por los cambios extremos de temperatura y humedad relativa, ya sea diaria como anual.

Bajo los principios de la llamada conservación preventiva, que considera esencialmente el tomar medidas que eviten o reduzcan el potencial de daño en términos globales (colecciones más que objetos) (Boletín GCI 1992: 5), se ha propuesto una planificación a mediano y largo plazo. 
Como uno de los principales objetivos planteados estaba el de realizar un acabado estudio de las condiciones medioambientales al interior de este museo, con el fin de: a) planificar la puesta en uso de los depósitos y otras dependencias en las cuales almacenar las colecciones; y b) llegar a definir líneas específicas de investigación respecto de la conservación de colección, hoy definida como de mayor riesgo (p.e., textiles, cestería, cuerpos humanos, papeles, maderas y otros).

La prevención asegurará a largo plazo la protección de las colecciones mediante un adecuado control de las condiciones ambientales (HR, temperatura, iluminación, desastres naturales, robos y mal trato de ellas, etc.) (Filden 1979: 10).

El control del ambiente físico, especialmente al interior de los museos, involucra aspectos tan importantes como el control de la luz, el que se constituye en uno de los agentes más dañinos y de difícil control. Asimismo las alteraciones o variaciones en la humedad y temperatura se constituyen en otro agente de deterioro (De Guichen 1988), ya sea al interior como al exterior de éste.

Otro aspecto de la mayor importancia lo constituye la adecuada documentación y posterior almacenaje de las colecciones (Azócar y Rodríguez 1988, 1989). Pero para que cualquiera acción de conservación preventiva que se asuma sea eficaz en lo que a una colección en particular se refiere, el conocimiento científico debe ser igualado a un decidido compromiso administrativo y así integrar esta tarea de conservación preventiva al funcionamiento regular de la institución (Boletín GCI 1992).

Existe plena conciencia en que el deterioro de las colecciones, especialmente arqueoantropológicas, se acelera y acrecienta en el mismo momento en que éstas son descubiertas y retiradas de una "matriz" que por mucho tiempo las ha cobijado (Stanley 1984). Es en este sentido que cobra valor el trabajo preventivo en la conservación de esos objetos, es decir, el aplicar todas aquellas medidas tendientes a disminuir los factores que puedan atentar contra la integridad de éstos. Se trata, entonces, de poseer un completo conocimiento de las condiciones climatológicas internas y externas (en terreno como en el museo), con el fin de asegurar la mayor estabilidad especialmente en términos de HR, temperatura, luz y transporte.
En el caso del edificio destinado al museo, la obtención de un control climático podrá dar seguridad a las colecciones, y eso se logra cuando hay pleno conocimiento de cada una de las unidades internas como externas. Sólo con esta base se podrán aplicar los correctivos necesarios para su estabilización (p.e., impermeabilización, aislación térmica, acceso controlado de luz, desinfección, acceso de polución, etc.). Sólo a partir de ese momento es prudente y aconsejable intervenir directamente las colecciones en términos de evaluar su estado actual de conservación, limpieza, desinfección, registro y eventuales restauraciones, etc.

La conservación del patrimonio cultural y natural es uno de los grandes desafíos a los que estamos enfrentados y debemos reconocer, que es el propio ambiente el agente más eficaz de deterioro de su propia obra y su entorno.

\section{Características generales del área}

El museo se ubica en el poblado de San Pedro de Atacama, localidad asentada en el margen noreste del Salar de Atacama, a unos $96 \mathrm{~km}$ al sureste de la ciudad de Calama y a 2436 m.snm.

Es el Padre Gustavo Le Paige quien por más de 25 años investigó y puso de relieve esta cultura ancestral a través de la formación de este museo que hoy en día es reconocido más allá de las fronteras nacionales.

Son escasos los ejemplos de instituciones que concentren en su propio territorio histórico la gran mayoría de las colecciones provenientes de la investigación antropoarqueológica y que concite atención científica y turística. Esto se ve resaltado por el hecho de instalarse en un territorio alejado de centros urbanos mayores, con una limitada comunicación (salvo estos últimos años) y en un medio geológico caracterizado como desierto de altura (Golubev y Reyes 1974), cuya principal característica es la alta variación climática (sobre todo térmica) diaria y anual, lo que hace difícil la instalación humana, dada la dura condición de vida. Esta "agresión" climática afecta sin duda la cantidad y variabilidad de recursos de todo orden.

\section{Características del edificio}

Ubicado a escasos metros de la plaza central del poblado de San Pedro de Atacama, presenta una difícil definición respecto de su orientación puesto 
que su planta general la constituyen tres octógonos unidos entre sí de manera triangular (Figura 1).

La construcción del mismo fue planteada en un terreno levemente elevado, vecino a la casa parroquial, y si bien es cierto fue concebido en su totalidad, su construcción se realizó en tres etapas $(1963,1972$ y 1978, respectivamente; Téllez 1992).

Su concepción constructiva (criticada por especialistas) mezcla fundamentos de concreto, murallas de adobes y pilares maestros de fierro como vigas de techumbre. Si bien estos elementos son incompatibles entre sí, hoy celebramos 30 años de funcionamiento ininterrumpido y los problemas derivados de la arquitectura son más bien calificados de menores, dado principalmente a que el grueso de la obra quedó inconcluso, faltando terminaciones generales tales como afinado y cubierta de pisos, aislación térmica y cielo, techumbre adecuada, revestimiento y pintura de muros.

El 100\% de las murallas (externas e internas) están construidas de adobes, alcanzando un espesor promedio de $45 \mathrm{~cm}$. Estos se presentan enlucidos con una mezcla de barro arcilloso en ambas caras. Interiormente se ha aplicado pintura látex en la totalidad del edificio, y sólo en el espacio destinado a los baños públicos se aplicó una cubierta de cemento en aquellos sectores más críticos (sector de instalación de artefactos sanitarios).

Si bien se puede anotar que el adobe es un material de características aislantes excepcionales, no menos cierto es el hecho de que éste actúa como "material tampón" (higroscópico) de altísima eficacia,

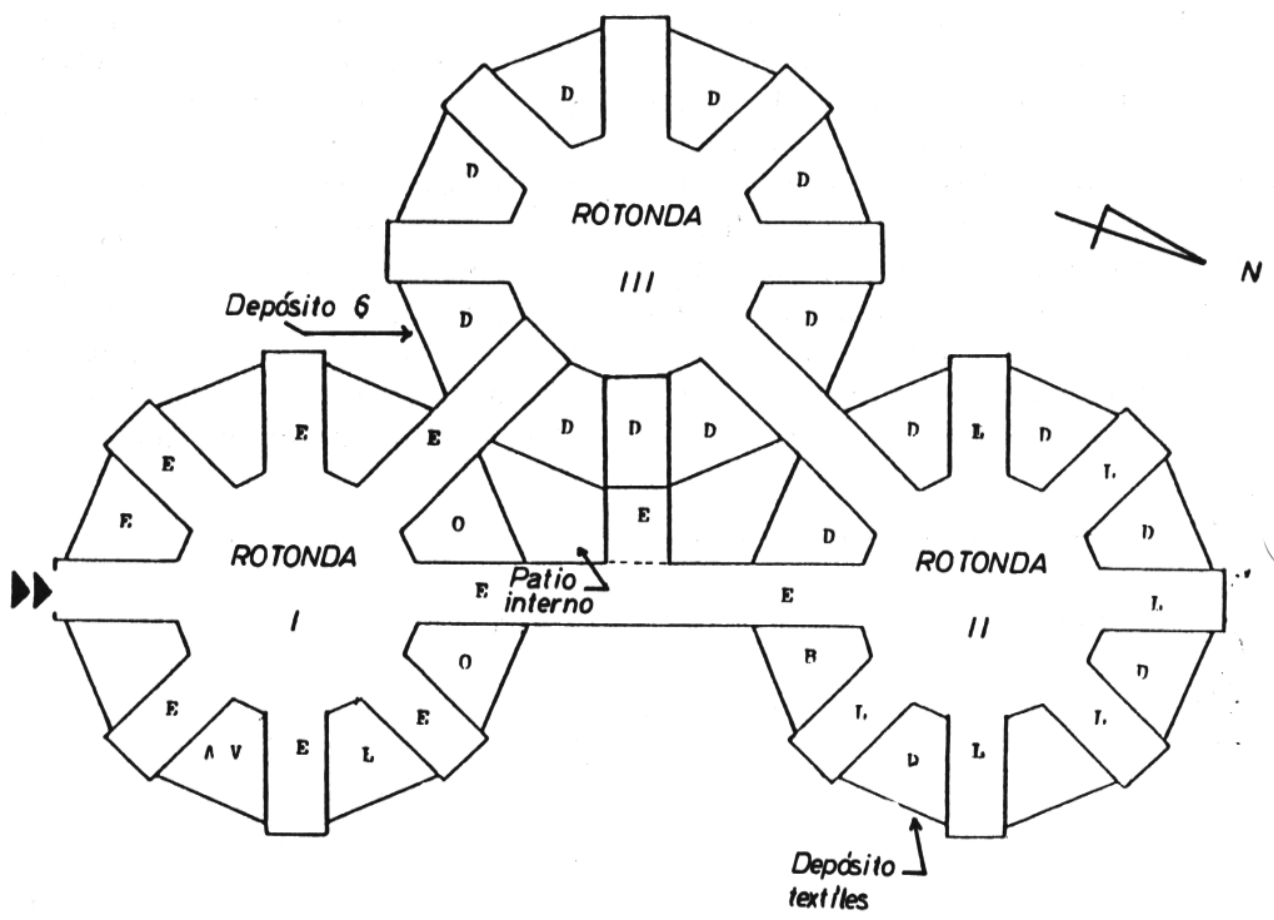

$5 \mathrm{mt}$.

\footnotetext{
E: Exhibición

O: Oficina

L: Laboratorio

B: Baño

D: Depósito

A-V: Audiovisual
}

Figura 1. Planta del Museo Arqueológico de San Pedro de Atacama. 
generando un problema adicional si no se controla de alguna manera (alta absorción de humedad). Si a esto se le adiciona el hecho de que en un $100 \%$ la aplicación de cielos rasos se ha efectuado con maderas aglomeradas (masisa), esta situación se acentúa. Actualmente un $70 \%$ de la superficie del edificio presenta un piso revestido con material plástico (flexit y linóleo), que le da una mejor aislación y facilidades en el aseo.

Uno de los mayores problemas está presente en la techumbre, pues la solución constructiva presenta un sinnúmero de caídas de agua, lo que hace difícil su control. A esta dificultad se le debe sumar el hecho de que en un $100 \%$ es de cubierta metálica (zinc acanalado), con los problemas fáciles de prever en un ambiente como el característico en estas regiones donde se registran amplísimas variaciones térmicas durante todo el año.

Otro factor coactuante en estas materias es la diversidad de formas que presentan los recintos, siendo una de las limitantes en un total y eficaz aprovechamiento de los espacios (nos referimos a los espacios destinados a depósitos), que presentan una forma de planta triangular, lo que en definitiva da un aprovechamiento espacial relativo (Téllez 1992), como asimismo amplios espacios destinados a pasillos subutilizados en la actualidad.

Bien es sabido que cualquier tipo de edificio requiere de un permanente cuidado y mantención para su óptima permanencia en el tiempo (Boletín GCI 1992). En el caso particular de edificios construidos en adobe, este cuidado debe ser más acucioso y periódico, puesto que está más expuesto al deterioro dada la frágil naturaleza de su material básico (barro arcilloso). Se ha podido constatar en el área que todos los problemas observados, ya sean filtraciones de aguas lluvia, polvo, etc., se deben casi con exclusividad al descuido y poca previsión por parte de los usuarios, atribuible quizás a la falta de recursos. Obviamente los costos de reparación, en estos momentos, han sido altos dado el avanzado estado de deterioro del inmueble, el que en poco tiempo demostrará daños irreversibles.

\section{Descripción del instrumental utilizado}

Para desarrollar el trabajo descrito se han utilizado tres termohigrógrafos a batería marca IZUSU de frecuencia semanal y para efectuar su calibración y control permanente se está utilizando un sicrómetro fijo, el que ofrece ventajas comparativas de fácil lectura frente a uno de molinete. Además se mantiene un set de 6 higrómetros de cabello dispuestos de manera permanente con el fin de apoyar un control visual rápido. Asimismo, se han confeccionado planillas especiales para registrar lecturas anotadas en años anteriores, al exterior del edificio y que corresponden a las realizadas por la estación local de Meteorología durante los años 1962, 1988, 1989 y 1990, respectivamente (Gráficos 1, 2, 3, 4 y 5), actualmente en la biblioteca de nuestra unidad (Le Paige 1962-63Ms; Escalante 1988Ms, 1989Ms).

\section{Análisis de gráficas de comportamiento climático ( $T^{\circ}$ y HR) en el Instituto de Investigaciones Arqueológicas y Museo}

Se ha tomado como punto de referencia la gráfica de comportamiento climático de un año, en un período del mes de agosto a agosto, que bien podría reflejar un "período tipo", representando un "valor promedio" comparable con otros años o períodos equivalentes. Asimismo se han definido cuatro puntos para la toma de datos, los que están caracterizados principalmente por su ubicación espacial y características diferenciales en términos constructivos. Como rango de comparación de dicho comportamiento climático se ha tomado gráfica (en igual período) de las condiciones ocurridas al exterior del edificio.

Los instrumentos fueron dispuestos y regulados al mismo tiempo con el fin de obtener datos comparables y ubicados generalmente en zonas más o menos similares:

\section{Patio interno (al aire libre).}

2. Dependencia interna del IIAM sin acondicionamiento térmico (Rotonda III, Depósito Antropología Física).

3. Dependencia interna del IIAM con acondicionamiento térmico (Depósito RII-D6, colección osteológica y otros).

4. Dependencia interna del IIAM con acondicionamiento térmico e introducción de humedad controlada (Depósito Rin-D1, colección de textiles).

Iguales mediciones se registran desde los meses de julio y agosto de 1991 en diversas dependencias del IIAM (laboratorios, depósitos, salas de exhibición, etc.) y en diferentes épocas del año, con el fin de evaluar el comportamiento integral del edificio durante las diferentes estaciones del año. 


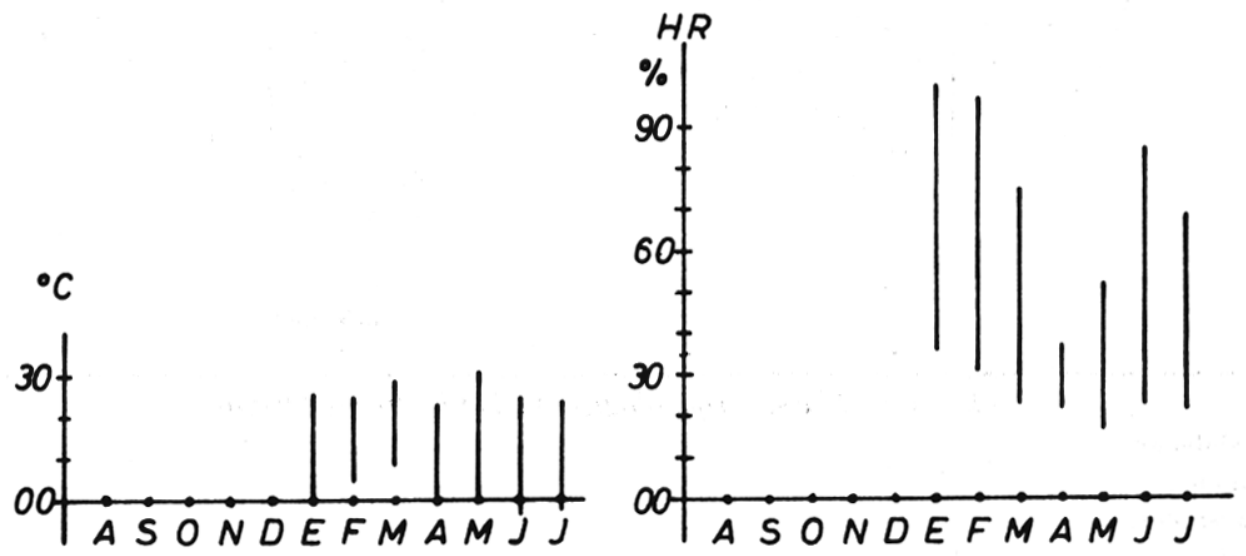

Gráfico 1. Patio Interno.
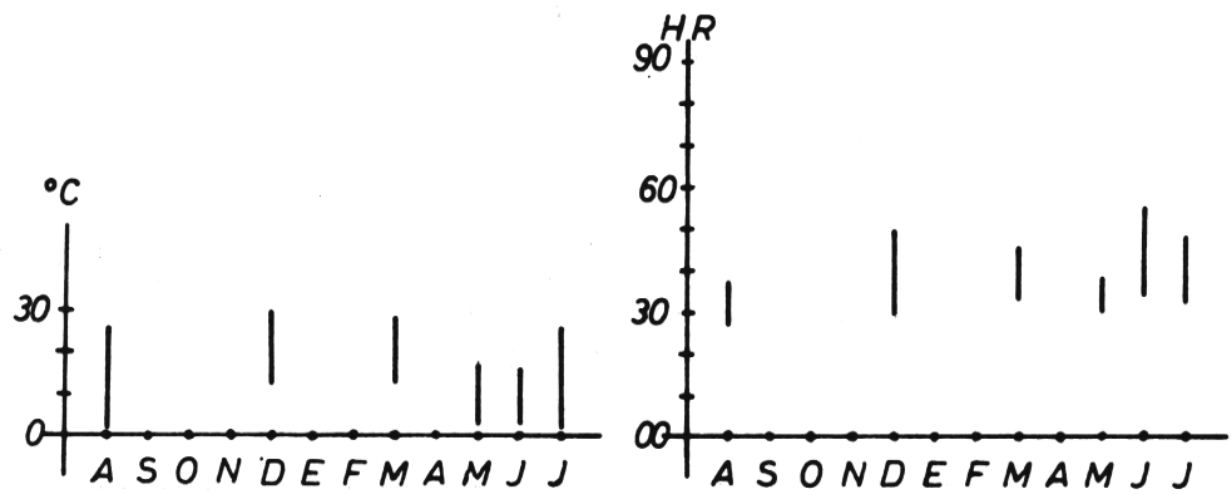

Gráfico 2. Depósito sin aislación térmica.

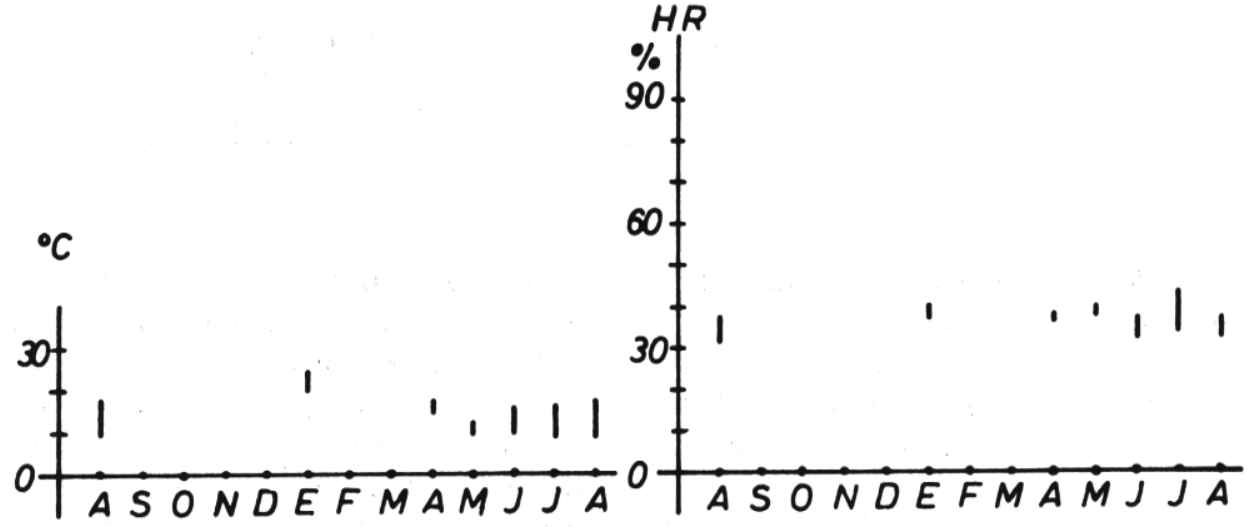

Gráfico 3. Depósito con aislación térmica. 


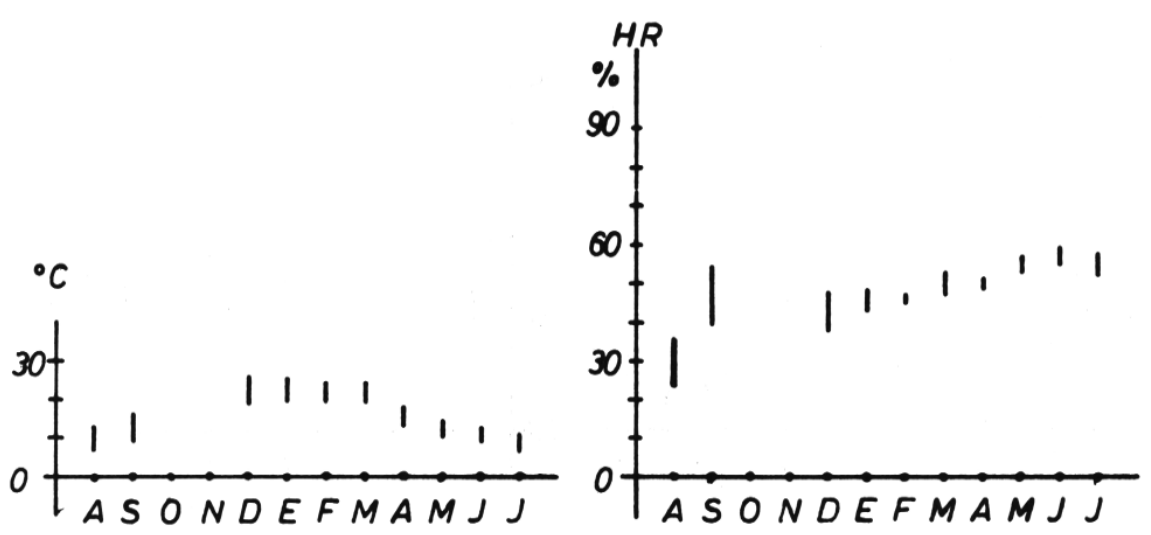

Gráfico 4. Depósito con aislación térmica y humedad controlada.
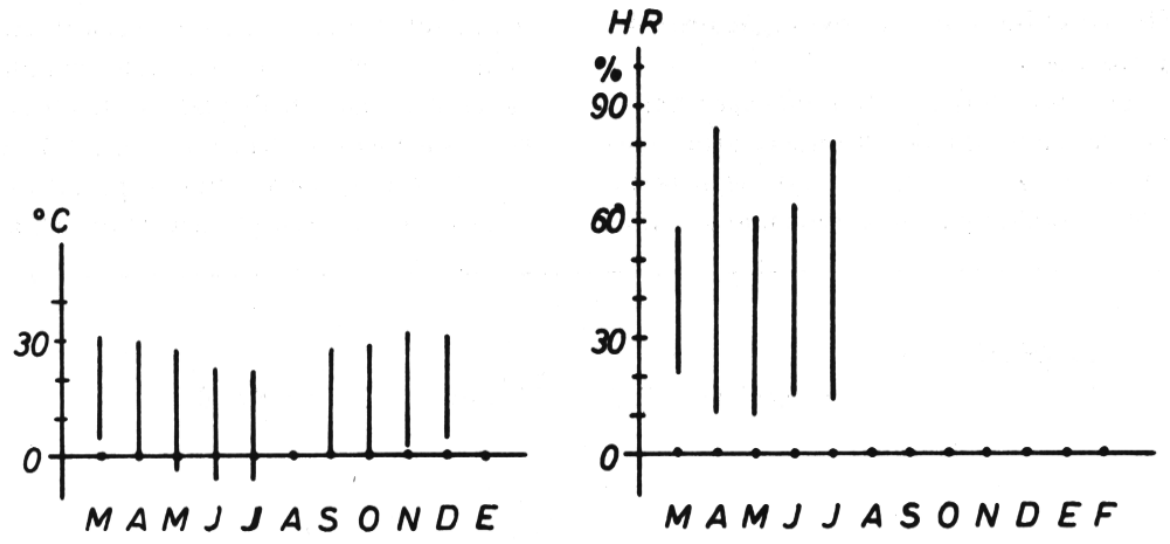

Gráfico 5. Año 1962.
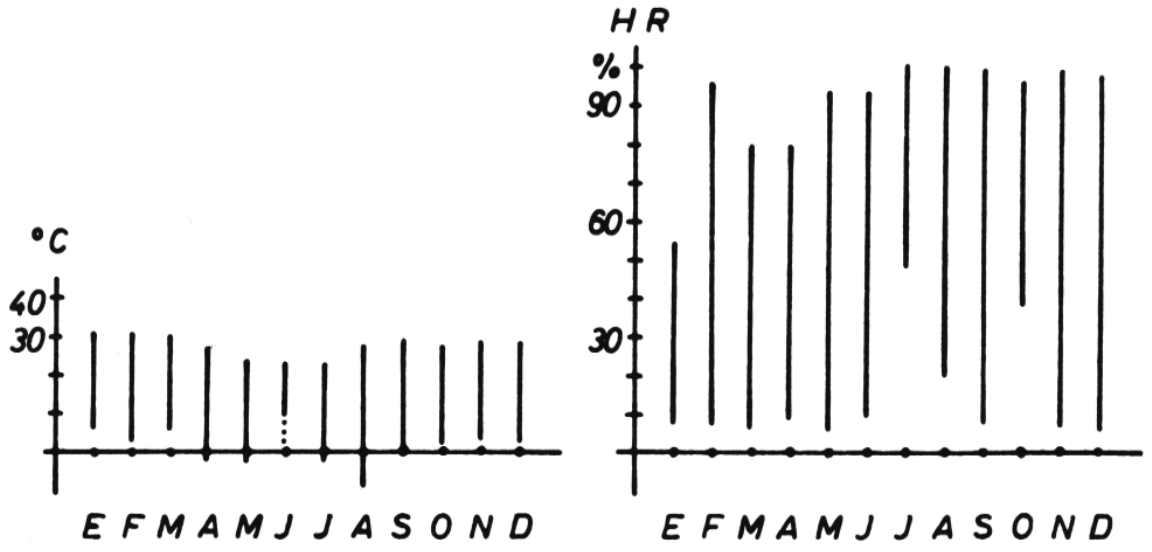

Gráfico 6. Año 1989. 


\section{REFERENCIAS CITADAS}

AZOCAR M., A. y A. RODRIGUEZ, 1988. Una experiencia en conservación de material arqueológico y etnográfico. Revista Museos 2: 12-13.

1989. Documentación de material arqueoetnográfico: Ficha básica y de embalaje. Museos 6: 12-14.

BOLETIN DEL GCI (The Getty Conservation Institute), 1992, 7 (1): 4-7.

DE GUICHEN, G, 1988Ms. Anotaçoes sobre umidade e temperatura nos Museus. Trad. AMICOM/77. Curso conservación preventiva en los Museos (Módulos, 1988).

ESCALANTE P., 1988Ms. Control climático, Estación San Pedro de Atacama, Departamento Hidrología, Sección Meteorología (octubre 1988-julio 1989). Archivo del IIAM, San Pedro de Atacama.

1989Ms. Control climático, Estación San Pedro de Atacama, Departamento Hidrología, Sección Meteorología (julio 1989 -julio 1990). Archivo del IIAM, San Pedro de Atacama.
FILDEN, B. M., 1979. Introducción a la conservación. UNESCO.

GOLUBEV G. y J. P. REYES, 1974. La zonación vertical geográfica en Chile entre $22^{\circ}-24^{\circ}$. Revista Geográfica 6 Año I: 1-8.

LE PAIGE G., 1962-63Ms. Libro diario de observaciones, Fuerza Aérea de Chile, Dirección de tránsito aéreo, Oficina Meteorológica de Chile, Estación San Pedro de Atacama. Archivo del IIAM, San Pedro de Atacama.

STANLEY, P., 1984. La conservación en excavaciones arqueológicas. ICCROM. Servicios Gráficos Colomina S.A., Roma.

TELLEZ C., 1992. El Museo Arqueológico de San Pedro de Atacama: Inicios de una conservación preventiva. Un compromiso con el futuro. Actas VII Jornadas Museológicas Chilenas, Santiago. 